\title{
A Case Report on Rolandic Epilepsy
}

\author{
Subbarayan Rajeswari ${ }^{1}$, Jayaprakash Jayapriyanjali ${ }^{2}$
}

\begin{abstract}
Benign focal epilepsy of childhood is an entity that includes characteristic clinical and electroencephalographic manifestations. An epileptic seizure is a transient occurrence of signs and/or symptoms due to abnormal excessive or synchronous neuronal activity in the brain. Epilepsy is a disorder of the brain characterized by an enduring predisposition to generate epileptic seizures and by the neurobiological, cognitive, psychological, and social consequences of this condition. Here we report a case of Rolandic epilepsy or benign childhood epilepsy with centrotemporal spikes (BCECTS), which is the most typical epilepsy, and the child presented with typical symptoms of active seizures in the form of uprolling of the eyes and jerky movements of the upper limbs.

Keywords: Epileptic seizure, Rolandic epilepsy.

Pondicherry Journal of Nursing (2021): 10.5005/jp-journals-10084-13109
\end{abstract}

\section{BACKGROUND}

Benign Rolando epilepsy or benign childhood epilepsy with centrotemporal spikes (BCECTS) is the most typical epilepsy. The sylvian epilepsy seizures are caused by the Rolando fissure in the brain (also known as the centrotemporal area, which is located around the Rolandic fissure and identified after Luigi Rolando). ${ }^{1}$

\section{Case Description}

A 4-year-old male child was apparently normal till $10 \mathrm{pm}$ at night, he woke up mid-sleep and had an episode of vomiting (not bloodstained, nonprojectile, containing food particles, nonbilious), which was followed by uprolling of the eye that is lasting for $\sim 1 / 2$ hour, after which he developed jerky movement of both upper extremities lasting for $\sim 5$ minutes, and then the child became drowsy and postictal drowsiness was present. The child was brought to casualty and got admitted to pediatric intensive care unit for further medical management. On examination, the child was in postictal drowsiness with uprolling of the eyes. Following investigations were performed to rule out the cause, such as magnetic resonance imaging (MRI) and electroencephalogram (EEG). The EEG report shows that centrotemporal spikes and sharp waves from both the cerebral hemispheres suggestive of generalized epileptic form discharges and the child was diagnosed with Rolandic epilepsy. The child was advised to continue the antiepileptic drugs - T. Lorazepam ( $5 \mathrm{mg} /$ twice in a day) and sodium valproate(4.5 mg/twice in a day).The child was hospitalized for 5 days, and parents were counseled regarding the child's condition, treatment, and management.

\section{Rolandic Epilepsy}

Benign Rolandic epilepsy (BRE), also called benign epilepsy with centrotemporal spikes (BECTS), is a type of childhood focal epilepsy characterized by motor focal seizures and specific EEG abnormalities in the absence of neurological deficits. Seizures begin in the Rolandic area of the brain, hence the name "Rolandic;" that is, the part of the brain that controls the face. Partial seizures start in a specific part of the brain and last for a brief span of time. The most common type of idiopathic epilepsy in children is BECTS.

\footnotetext{
1,2Department of Child Health Nursing, Kasturba Gandhi Nursing College, Puducherry, India

Corresponding Author: Subbarayan Rajeswari, Department of Child Health Nursing, Kasturba Gandhi Nursing College, Puducherry, India, Phone: +91 9442254624, e-mail: rajeswaris@kgnc.ac.in

How to cite this article: Rajeswari S, Jayapriyanjali J. A Case Report on Rolandic Epilepsy. Pon J Nurs 2021;14(2):48-50.

Source of support: Nil

Conflict of interest: None
}

The term "idiopathic" has been replaced with "genetic" in the most recent International League Against Epilepsy classification proposal. However, because the genetic marker in children with $\mathrm{BRE}$ is unknown, it is most likely genetic (Fig. 1). ${ }^{2-4}$ Incidence:

- Benign Rolandic epilepsy can start anytime between the ages of 1 to 14 years.

- BRE occurs more often in male than in female with a 1.5 to 1 predominance.

- The incidence of BRE is 10 to 20 per 100,000 children up to age 15 years. BRE makes up to $15 \%$ of all epilepsy cases in children, which makes it the most common epilepsy syndrome of childhood. Adults are not affected. ${ }^{5}$

Causes:

BRE is thought to be a genetic disorder, an autosomal dominant inheritance. Studies suggest that certain regions on chromosomes 11 (11p13) and chromosome 15 (15q14) may be involved in BRE, but a specific gene has not been identified. ${ }^{6}$

\section{Clinical Manifestation}

Infrequent, often single, focal seizures consisting of unilateral facial sensorimotorseizures are the hallmarks of Rolandic epilepsy.

Are frequently contained entirely within this lower lip or spread to the ipsilateral hand. Sensorimotor manifestations are clonic contractions that occur suddenly, continuously, or in bursts, usually lasting a few seconds to a minute. 
Oropharyngolaryngeal ictal manifestations: Inside the mouth, there are sensorimotor symptoms. Numbness and, more commonly, parenthesis (tingling, prickling, and freezing) are usually unilateral or, in rare cases, highly localized to a single tooth.

Arrest of speech is a form of anarthria: The child is unable to speak a single word and communicates only through gestures.

Excessive salivation: A prominent autonomic manifestation is frequently associated with unifacial seizures, oropharyngolaryngeal symptoms, and speech arrest.

Consciousness and recollection: They are not affected and able to sustain in more than half $(58 \%)$ of Rolandic seizuresd rowsiness.

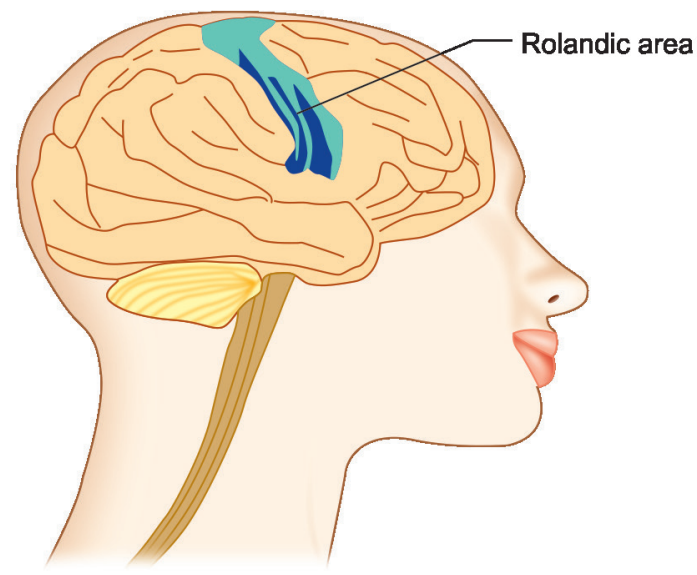

Fig.1: Anatomical part of Rolandic
Progression to unilateral convulsions or generalized tonic-clonic seizuresoccurs in around half of the children, and hemiconvulsions may be followed by postictal Todd's hemiparesis.

Syncope-like epileptic seizures: They may probably occur as a concurrent symptom of Panayiotopoulos syndrome.

Duration and circadian distribution: Rolandic seizures are usually short-lived, lasting only 1-3 minutes. Three-quarters of seizures happen during nonrapid eye movement sleep, usually at the start of the sleep cycle or just before waking up.

Atypical forms: Atypical manifestations of Rolandic epilepsy include early onset, developmental delay, or learning difficulties at inclusion, other seizure types, and atypical EEG abnormalities. ${ }^{7-10}$

\section{DiAgnosis}

- EEG spikes in the centrotemporal regions of the brain in children with CECTs. These findings aid in the confirmation of the diagnosis.

- MRI: In children with CECTS, this test is usually normal. When the history and EEG are both very typical, it is rarely helpful.

\section{Nursing Care}

Epilepsy can be difficult to manage. If a seizure starts as only a warning, nursing management should be the same as for the simple partial seizure (Fig. 2).

- Assess the child for any signs of injury. Check for limb displacement, as this may indicate the child has a fracture.

- Educate the parents to watch for any behavioral changes in the child.

- Talk in a calm and reassuring voice and communicate with the child throughout the seizure.

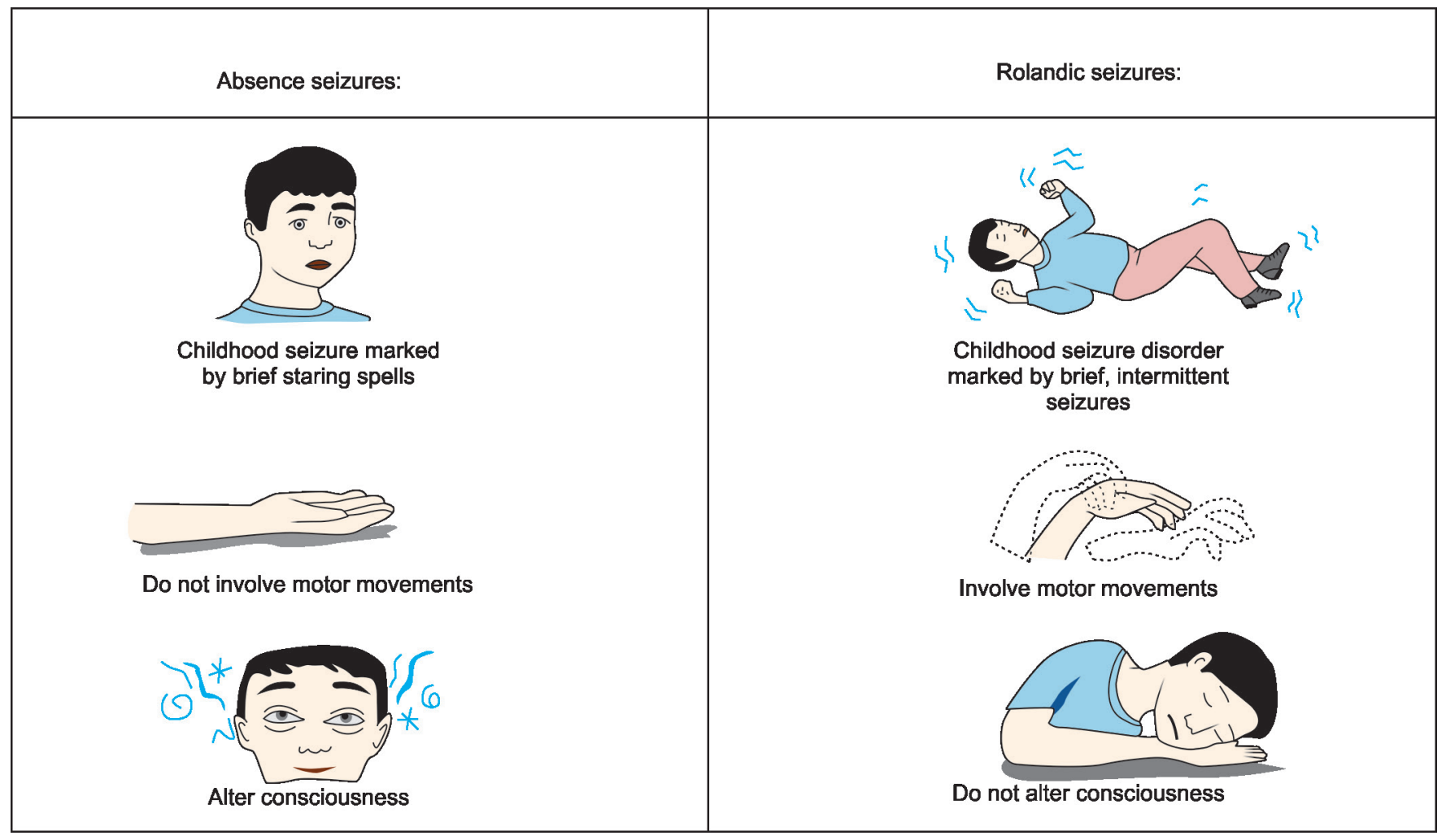

Fig.2: Clinical features of Rolandic epilepsy 
- Educate the mother to provide a ketogenic diet, which contains (high fat with low carbohydrates and controlled protein (egg, whole dairy products, pulses, cereals, fruits, and dried fruits).

- Counsel the parent about the child's health conditions and further prognosis.

- Encourage the parents to interact with the child while giving any activities.

- Educate the mother about the first aid for the seizure episodes.

\section{Prognosis}

The child's prognosis was better, after the medical management and further close monitoring; the childwas not having any complaints of seizure episodes. Child activity was good. He started interacting with his parents and healthcare team members. Then the child was shifted to the pediatric ward for 1-day observation. The child was apparently normal, no complications occur. The child's parents were counseled by doctors about the child's present condition and they had planned to discharge the child at home. Parents were educated by the staff nurse about the medications, follow-up, and diet for the child. If further episodes of any seizure, they were advised to immediately bring the child to the hospital.

\section{ConcLusion}

BRE is a developmental condition, meaning that it evolves as the child matures. Certain symptoms completely resolve, like the seizures and the EEG abnormality. One hundred percent of children "grow out" of their seizures by the age of sixteen years. The EEG usually goes back to normal at around the same time, indicating that the tendency for recurrent seizures remains, about 1 to $2 \%$ over a lifetime. ${ }^{11}$ The treatment goal of the child with Rolandic epilepsy is the prevention of further complications. This is infrequent and harmless, and virtually all children outgrow the condition. These children usually have normal intelligence and development.
It would be resolved by puberty. Appropriate counseling and identification will help to prevent the epilepsy.

\section{References}

1. Crino PB. M TOR signaling in epilepsy: insights from malformation of cortical development.Cold Spring Harb Perspect Med2015;5(4):22442. DOI: 10.1101/cshperspect.a022442.

2. Dalla Bernardina B. Idiopathic partial epilepsies in children. In: Roger J, Bureau M, Dravet C, et al., editors. Epileptic syndromes in infancy, childhood and adolescence. London: John Libbey Ltd., 1992.

3. Dibbens LM, Mullen S, Helbig I, et al. Familial and sporadic $15 q 13.3$ microdeletions in idiopathic generalized epilepsy: precedent for disorders with complex inheritance. Hum Mol Genet 2009;18(19):3626-3631. DOI: 10.1093/hmg/ddp311.

4. Tassinari CA, Meletti S, Rubboli G, et al. Acquired epileptic aphasia. Epilepsy Sleep 2001; 2001:173-190. https://doi.org/10.1016/S13882457(00)00408-9

5. Neubauer BA, Fiedler B, Himmelein B, etal. Centrotemporal spikes in families with Rolandic epilepsy: linkage to chromosome $15 q 14$. Neurology 1998;51(6):1608-1612. DOI: 10.1212/WNL.51.6.1608.

6. Beaussart M. Benign epilepsy of children with Rolandic (centrotemporal) paroxysmal foci. A clinical entity. Study of 221 cases. Epilepsia13(6): 795-811. DOI: 10.1111/j.1528-1157.1972.tb05164.x

7. Loiseau P, Beaussart M. The seizures of benign childhood epilepsy with Rolandic paroxysmal discharges. Epilepsia14(4): 381-389. DOI: 10.1111/j.1528-1157.1973.tb03977.x.

8. Lerman P, Kivity S. Benign focal epilepsy of childhood. A follow-up study of 100 recovered patients. Arch Neurol32(4): 261-264. DOI: 10.1001/archneur.1975.00490460077010.

9. Panayiotopoulos CP. Benign childhood epilepsy with centrotemporal spikes or Rolandic seizures. In: Benign childhood partial seizures and related epileptic syndromes. London: John Libbey Eurotext, pp.33-100.

10. https://www.epilepsy.com/learn/types-epilepsy-syndromes/ childhood-epilepsy-centrotemporal-spikes

11. Dimassi S, Labalme A, Lesca G, et al. A subset of genomic alterations detected in Rolandic epilepsies contains candidate or known epilepsy genes including GRIN2A and PRRT 2. Epilepsia 2014;55(2):370-378. DOI: 10.1111/epi.12502. 